\title{
Research Progress on the Effect of Massage on Immune System Function in Children
}

\author{
Yameng $\mathrm{Xu}^{1}$, Lin $\mathrm{Wu}^{2}$, Fangfang Yang1, Zhengrong $\mathrm{Ye}^{1 *}$ \\ ${ }^{1}$ Shaanxi University of Traditional Chinese Medicine, Xianyang 712046, Shaanxi Province, China \\ ${ }^{2}$ Xian Yang Central Hospital, Xianyang 712000, Shaanxi Province, China \\ *Corresponding author: Zhengrong Ye, 474877278@qq.com
}

\begin{abstract}
As a traditional Chinese medicine treatment, massage has significant clinical effect. In recent years, a large number of clinical studies have shown that the mechanism of massage in the treatment of diseases is related to the regulation of immune function. In this article, we review the existing problems and prospects of massage on children with diarrhea, asthma and recurrent respiratory tract infection in relation to its effect on the regulation of immune function.
\end{abstract}

Keywords: Infantile massage; Immunity

Publication date: July 2021; Online publication: July 30, 2021

\section{Introduction}

As a non-drug therapy, massage can dredge meridians, promote $q i$ and blood circulation, and regulate yin and yang. This therapy does not only directly acts on the meridians, acupoints and some specific parts of the body surface to produce mechanical effects, such as relaxing tendons and activating collaterals, relieving spasm and fatigue, and promoting circulation ${ }^{[1]}$, but also plays an important role in the influence of neuroendocrine immune network system ${ }^{[2]}$. Focusing on the effect on immune system function, it is of great value to study the mechanism of infantile massage for its further application.

\section{Effect of infantile massage on immune system function of children with diarrhea}

The main manifestations of infantile diarrhea are increased frequency of bowel movements and thin feces. Some studies believe that low immune function is an important cause of the disease ${ }^{[3]}$. Xiang and Zhou found that infantile massage can improve the concentration of $\operatorname{IgA}$ and $\operatorname{IgG}$ in children with diarrhea ${ }^{[4]}$. Wang et al. found that tonifying the large intestine, spleen meridian, pushing the three passes and rubbing the abdomen clockwise can reduce the frequency of defecation, improve the stool characteristics, and increase the $\mathrm{IgA}, \mathrm{IgG}$, and $\mathrm{CD} 4^{+} / \mathrm{CD}^{+}$concentration in children ${ }^{[5]}$. Wang carried out massage using two anti-diarrhea paste on 40 children with diarrhea, and found that $\mathrm{IgG}, \operatorname{IgA}$ and $\mathrm{CD} 4^{+} / \mathrm{CD}^{+}$concentrations in children were significantly increased ${ }^{[6]}$.

\section{Effect of infantile massage on the immune system function of children with asthma}

Asthma is a kind of infantile disease caused by external pathogenic factors, such as phlegm qi obstruction and lung failure. Infantile massage plays an important role in improving the clinical efficacy of infantile asthma. Ma applied massage to 80 children with cough variant asthma ${ }^{[7]}$. After 3 courses of treatment, the levels of $\operatorname{IgA}$ and $\operatorname{IgG}$ increased. Tian et al. found that infantile massage can effectively improve children's $\mathrm{CD}^{+}, \mathrm{CD}^{+}, \mathrm{CD}^{+}, \mathrm{CD}^{+}, \mathrm{CD} 25^{+}, \mathrm{CD}^{+} \mathrm{CD} 25^{+} \mathrm{Foxp}^{+}$, transforming growth factor $\beta 1$ (TGF- $\left.\beta 1\right)^{[8]}$. At the same time, the levels of histamine, histamine receptor $\mathrm{H} 1$ (h1r), histamine receptor $\mathrm{H} 2(\mathrm{H} 2 \mathrm{R})$ and 
leukotriene C4 (LTC4), leukotriene D4 (LTD4), and leukotriene E4 (LTE4) in peripheral blood and lymphocytes were significantly lower than those before treatment ${ }^{[9]}$. Li et al. found that infantile massage can reduce the concentration of interleukin (IL)-17, IL-33 and IL-6 in serum ${ }^{[10]}$.

\section{Effect of infantile massage on immune system function of children with recurrent respiratory tract infection}

Recurrent respiratory tract infection refers to the frequent occurrence of upper and lower respiratory tract infection within one year, which is beyond the normal range, in which the immune status of children is the key factor of the disease ${ }^{[11]}$. Zhuoyue found that the immune function of children with recurrent respiratory tract infection can be effectively improved by chiropractic therapy, the total clinical effective rate was about $89.50 \%$, and the content of serum immune indexes $\left(\operatorname{IgA}, \operatorname{IgM}, \mathrm{CD}^{+}, \mathrm{CD}^{+}\right)$was significantly improved ${ }^{[12]}$. Li et al. found that chiropractic massage combined with kneading Dazhui, Feishu, Pishu, Shenshu and other acupoints can improve the serum $\mathrm{CD}^{+}$and $\mathrm{CD}^{+}$levels in children ${ }^{[13]}$. Chen also found that infantile massage can improve the level of $\mathrm{IgG}$ and $\mathrm{IgA}$, and improve the immunity of children ${ }^{[14]}$.

The mechanism of infantile massage in the treatment of diseases may be related to its influence on the immune function of children. The current research pertains to the application of different massage techniques, operation sites and operation time in the treatment of the same disease, but the related immune indexes used in these studies are not comprehensive and systematic. In addition, there are few studies on the immunomodulatory mechanism of infantile massage through animal experiments. In the future research on the mechanism of infantile massage regulating immune function, first of all, we should focus on the massage treatment of specific diseases, formulate and standardize an effective massage technique, operation site and operation time through evidence-based medicine. Secondly, we should focus on the concept of holism and balance and pay attention to the correlation between contradictions in the theory of traditional Chinese medicine and immunology. Thirdly, we should investigate this aspect through animal experiments using treatment in line with the characteristics of infantile massage therapy.

\section{Funding}

Shaanxi Provincial Department of Technology Social Development Project (2018SF-303)

\section{Disclosure statement}

The authors declare that there is no conflict of interest.

\section{References}

[1] Guo Z, 2009, Direct Stimulation and Local Response: One of the Basic Physiological Principles of Massage Therapy. Guide of Traditional Chinese Medicine, 15(9): 31-33.

[2] Li D, 2005, Clinical Immunology. Fudan University Press, Shanghai, 25.

[3] Lei J, Wang D, Li J, et al., 2016, Liu's Infantile Massage "Tui Wu Jing" as the Main Treatment for Infantile Diarrhea of Spleen Deficiency Type. Massage and Rehabilitation Medicine, 7(01): 37-40.

[4] Xiang S, Zhou S, 2019, Effect of Traditional Chinese Massage Combined with MAMIAI on Infantile Diarrhea and Immune Function. China Maternal and Child Health Care, 34(3): 686-689.

[5] Wang J, Liu L, Sun Z, et al., 2019, Therapeutic Effect of Jianpi Zhixie Decoction Combined with Massage on Infantile Diarrhea and its Influence on Immune Function. Chinese Journal of Traditional Chinese Medicine, 37(2): 400-402.20. 
[6] Wang X, 2018, Effect Analysis of Erzhu Zhixie Plaster Combined with Infantile Massage in the Treatment of Infantile Diarrhea and its Influence on Immune Indexes. Pediatrics of Integrated Traditional Chinese and Western Medicine, 10(3): 242-245.

[7] Ma Y, 2017, Effect of Massage on Airway Function and Immune Function in Children with Cough Variant Asthma. Journal of Community Medicine, 15(19): 4-6.

[8] Tian F, Li Q, Cui J, et al., 2014, Effect of Massage on TLRs Expression of Inflammatory Cells in Children with Asthma in Chronic Remission. China Maternal and Child Health Care, 29(21): $3512-$ 3514.

[9] Tian F, Li Q, Cui J, et al., 2014, Effect of Infantile Massage on the Levels of Histamine, Receptor and Leukotriene in Peripheral Blood of Asthmatic Children. China Maternal and Child Health Care, 29(24): 3987-3989.

[10] Li Q, Tian F, Cui J, et al., 2014, Effect of Infantile Massage on the Levels of IL-17, IL-33, IL-6 in Children with Asthma in Different Periods. China Maternal and Child Health Care, 29(04): 530-532.

[11] Zhu X, Yin W, Jiang H, et al., 2010, Levels of Serum Immunoglobulin, IgG Subclass and Cellular Immunity in Children with Recurrent Respiratory Tract Infection. Journal of Clinical Pediatrics, 28(02): 135-137.

[12] Zhuoyue, Zhang X, Liu M, et al., 2013, Effect of Chiropractic Therapy on Immune Function of Children with Recurrent Respiratory Tract Infection. China Maternal and Child Health Care, 28(23): 3782-3783.

[13] Li G, Chen Y, Chen X, 2017, Effect of Infantile Massage on Physical Development and Immune Function of Kindergarten Children. Shenzhen Journal of Integrated Traditional Chinese and Western Medicine, 27(13): 70-71.

[14] Chen W, 2017, Effect of Infantile Massage on Serum Immunoglobulin Level in Children with Recurrent Respiratory Tract Infection. Guangming Traditional Chinese Medicine, 32(20): 2976-2978. 